\title{
Early and Late Assessment of Left Ventricular Function Using Global Longitudinal Strain After Primary Percutaneous Coronary Intervention
}

\author{
Said Shalaby Montaser ${ }^{1}$, Mohamed Fahmy Elnoamany ${ }^{1}$, Abdelrahman Hassan Elbokary ${ }^{2}$, \\ Amr Yosry Emam, * \\ ${ }^{1}$ Cardiology Department, Menoufia University, Menoufia, Egypt \\ ${ }^{2}$ National Heart Institute, Giza, Egypt \\ ${ }^{3}$ Aswan Heart Center, Aswan, Egypt
}

Email address:

mnoamany@hotmail.com (M. F. Elnoamany), amryosry26@gmail.com (A. Y. Emam)

${ }^{*}$ Corresponding author

\section{To cite this article:}

Said Shalaby Montaser, Mohamed Fahmy Elnoamany, Abdelrahman Hassan Elbokary, Amr Yosry Emam. Early and Late Assessment of Left Ventricular Function Using Global Longitudinal Strain after Primary Percutaneous Coronary Intervention. Cardiology and Cardiovascular Research. Vol. 4, No. 4, 2020, pp. 180-186. doi: 10.11648/j.ccr.20200404.13

Received: September 19, 2020; Accepted: September 30, 2020; Published: October 13, 2020

\begin{abstract}
This prospective cohort study was conducted on fifty patients presented with STEMI and underwent primary PCI to Assess the impact of primary percutaneous coronary intervention (Primary PCI) on the left ventricular (LV) systolic function in patients with Acute STEMI using speckle tracking Echocardiography within the first 24 hrs and within 1 month after performing PCI. All patients were evaluated by history taking, clinical examination, laboratory investigations and coronary angiography assessment with revascularization of the infarct-related artery (IRA). All patients had CCU admission for 3 days. Conventional 2D echocardiography was performed within $24 \mathrm{~h}$ of Primary PCI to assess LV Global longitudinal peak systolic strain (GLPSS). All patients had been discharged to home with the guidelines-based medical treatment including (DAPT, Atorvastatin, ACEIs, Beta blockers). GLPSS was re-assessed after 1 month. The patients were divided into two groups: improved and non-improved, according to the improvement of LV systolic function measured by GLPSS. Improvement is defined by increase of GLPSS $\geq 10 \%$. Our study demonstrated Improvement of LV function was based on GLPSS and was observed in 54\% of the patients. Peak cardiac troponin T level, Peak creatine phosphokinase levels (CPK), LV diastolic function, and baseline GLPSS were identified as independent predictors of recovery of LV function. The patients who showed improvement of GLPSS were associated with improvement of EF (using simpson method).
\end{abstract}

Keywords: Acute Myocardial Infarction, Left Ventricular Function, Longitudinal Strain, Speckle Tracking Echocardiography

\section{Introduction}

For decades, two-dimensional (2D) and Doppler echocardiography were the central pillars of evaluating left ventricular (LV) function. For purely practical reasons, many clinicians still resort to measurements of LV ejection fraction (EF) as well as the visual analysis of myocardial wall motion when they evaluate LV global and regional performance. However, these methods have a significant inter-observer variability as they depend on the skills and experience of the user. The undisputed need for simple, readily accessible and reliable methods for evaluating function has driven industry towards the development of semi- or fully- automated methods and post-processing tools for quantifying LV function [1]. The European Association of Cardiovascular Imaging (EACVI) and the American Society of Echocardiography (ASE) acknowledged the additional value of deformation measurements over traditional functional parameters, such as LV EF, and recommended the technique 
now for clinical use [2]. The present study aimed to assess the GLPSS by Speckle Tracking Echocardiography (STE) in patients with STEMI in the first $24 \mathrm{hrs}$ after primary PCI and follow-up within 1 month to assess the impact of Primary PCI on the LV systolic function and detect the prognostic predictors of improvement.

\section{Material and Methods}

This prospective cohort study was conducted on fifty patients their ages ranged from 31 to 73 years with mean of (52.82 \pm 10.74$)$. Male gender accounts 90\% underwent primary PCI within a time period from April 2019 to March 2020 after approved by the ethical committee Faculty of Medicine and informed written consent was taken from patients.

\subsection{Inclusion Criteria}

Patients, with STEMI with symptoms onset less than $12 \mathrm{~h}$, who underwent successful primary PCI, were included. Diagnosis of acute myocardial infarction was made on the basis of typical ECG changes and ischemic chest pain associated with elevation of cardiac biomarkers.

\subsection{Exclusion Criteria}

Patients with following conditions were excluded from the current study: preexisting cardiomyopathy, moderate and severe valvular heart disease, renal impairment, congenital heart diseases and poor echocardiographic windows.

\subsection{Clinical Investigation}

On admission, all patients were subjected to the following: (i) taking full history, (ii) full systemic examination for other associated medical or surgical problems, (iii) standard 12-lead ECG using three-channel direct writing recorder with a paper speed $25 \mathrm{~mm} / \mathrm{s}$ and standardization adjusted to $1 \mathrm{~mm} / \mathrm{mv}$ (ECG was done on admission and repeated every six hours in the first $24 \mathrm{~h}$; thereafter, daily during the hospital course), (iv) laboratory investigation including estimation of cardiac enzymes (CPK, $\mathrm{CK}-\mathrm{MB}$, and Troponin T) (on admission, post intervention six hourly in the first $24 \mathrm{~h}$, and then once daily till normalization), coagulation profile, kidney function tests, liver function tests, and serum electrolytes including serum sodium and potassium.

\subsection{Echocardiography Study}

Echocardiography was performed within $24 \mathrm{~h}$ after primary PCI including conventional parameters: end diastolic dimension and volume (LVIDd, and EDV), end systolic dimension and volume (LVIDs and ESV), Fractional shortening (FS), EF, septal and posterior wall thickness (LVSWT and LVPWT), E/A, E/e'. Patients were imaged in the left lateral decubitus position using a commercially available system. [(Vivid E9, General Electric-Vingmed, Horton, Norway) at Menoufia University - Department of Cardiology and (Vivid T8, General Electric-Vingmed, Horton, Norway) at Elzaitoun Specialized Hospital].

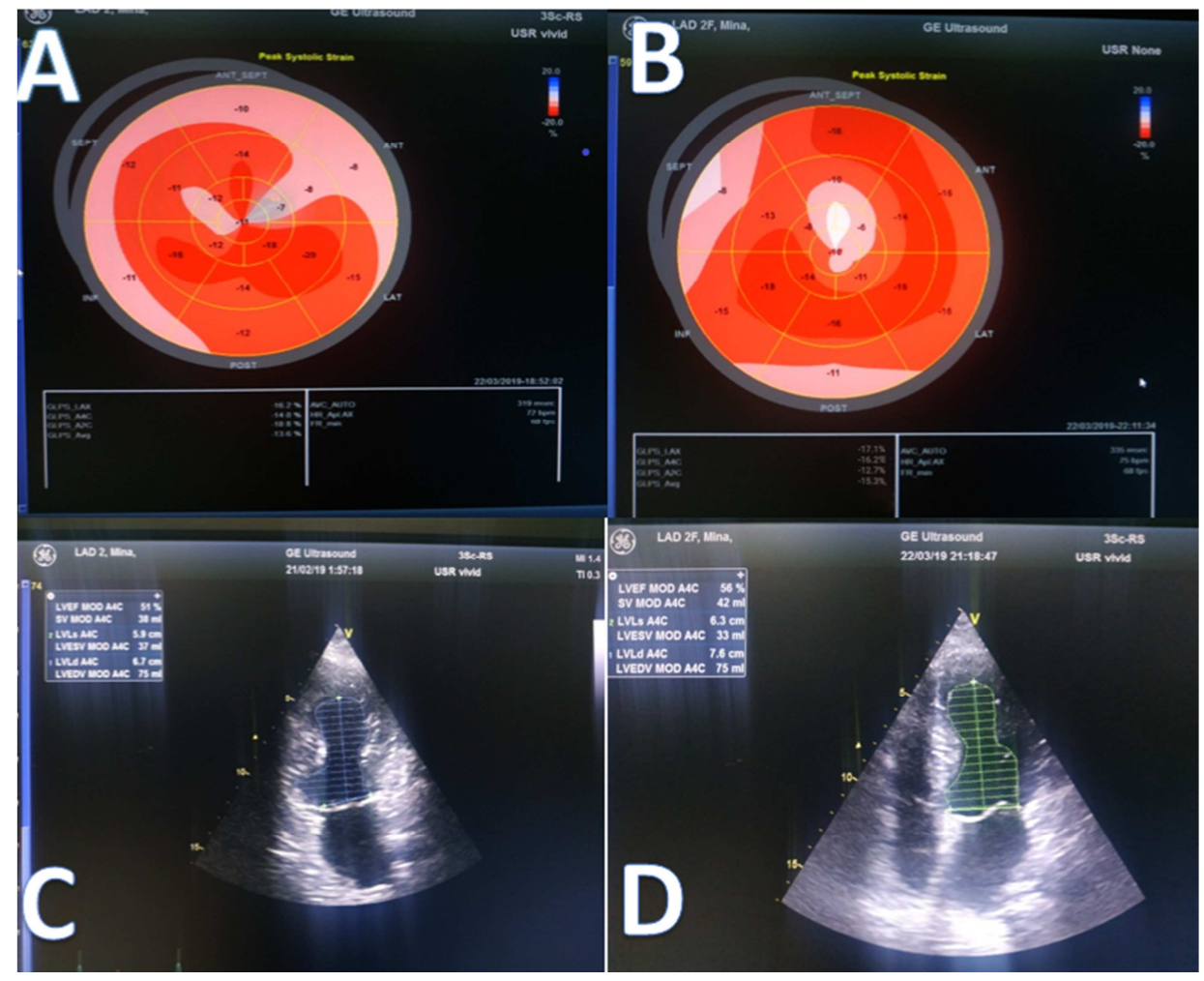

Figure 1. GLPSS and EF of representative patient from improved group at the baseline and follow-up studies. (A) Bull's-eye plots at the baseline echocardiography study. (B) Bull's-eye plots at the follow-up echocardiography study. (C) Simpson method measuring EF at the baseline echocardiography. (D) Simpson method measuring EF at the follow-up echocardiography. 


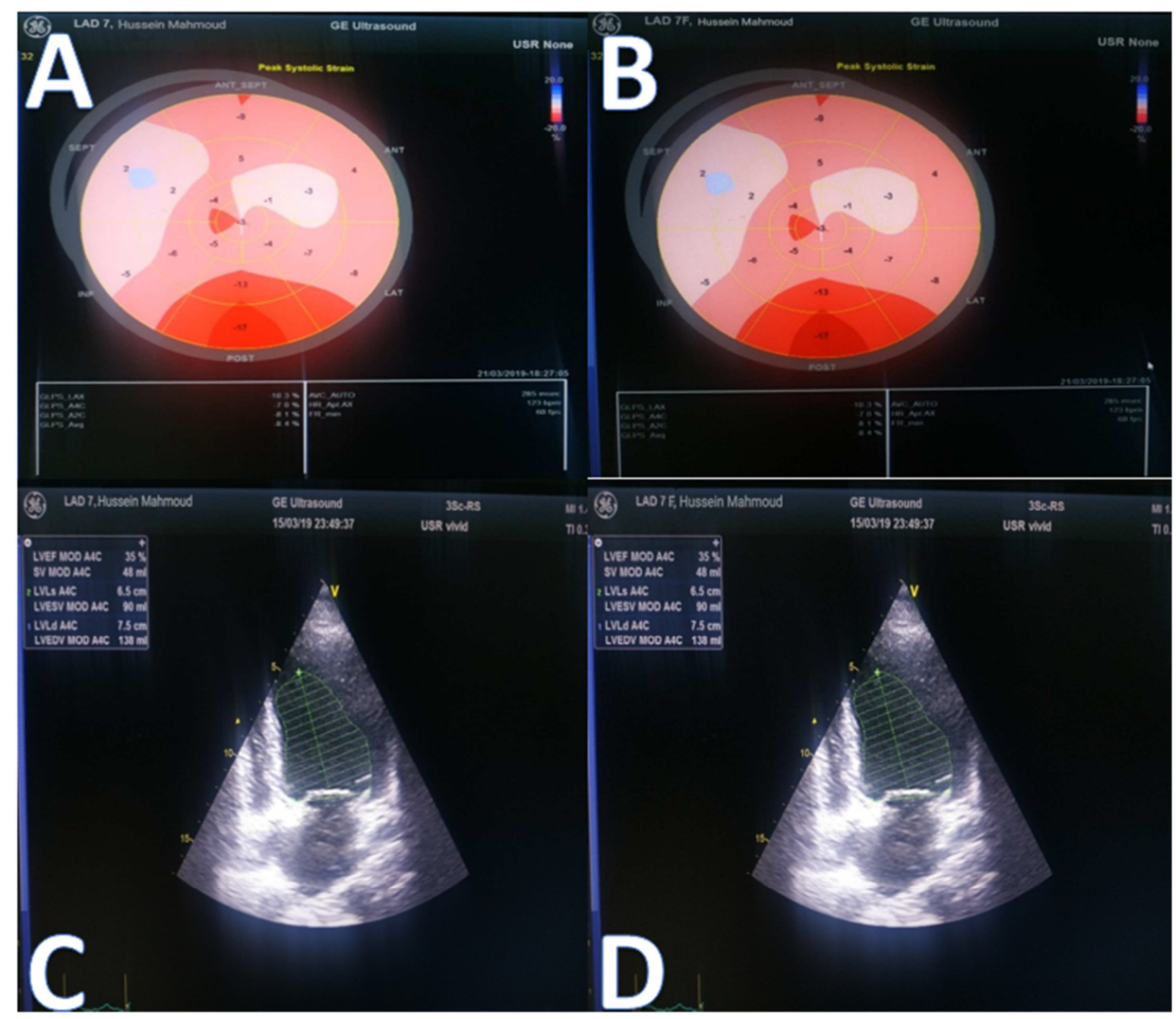

Figure 2. GLPSS and EF of representative patient from non-improved group at the baseline and follow-up studies. (A) Bull's-eye plots at the baseline echocardiography study. (B) Bull's-eye plots at the follow-up echocardiography study. (C) Simpson method measuring EF at the baseline echocardiography. (D) Simpson method measuring EF at the follow-up echocardiography.

\subsection{GLPSS Using Automated Function Imaging}

Apical four- and two-chamber views as well as longaxis views were used for quantification of GLPSS by automated function imaging speckle-tracking analysis. All analysed images were recorded with a frame rate of at least $40 \mathrm{fps}$ for reliable analysis by the software. First, the LV end-systolic frame was defined by determining the closure of the aortic valve in the apical long-axis view. Then the time interval between R-wave and aortic valve was automatically measured and used as a reference for the four- and two-chamber views. After defining the mitral annulus and LV apex with three index points in all three apical views, the LV endocardial border was automatically traced at end-systole and the created region of interest was manually adjusted to the thickness of the myocardium. Tracking quality was validated in all segments from the three apical views. Segments which failed to track by the software were manually adjusted by the operator. Any segments which subsequently failed to track were automatically discarded by the software for the calculation of global strain. Analysis was feasible in $97 \%$ of the segments. Global longitudinal peak systolic strain for the complete LV was provided by the software using a 17 segment model in a 'bull's eye' plot calculated as the average of longitudinal peak systolic strain of each view (Figures 1-2).
All patients had CCU admission for 3 days then discharged to home with the guidelines-based medical treatment including (DAPT, Atorvastatin, ACEIs, Beta blockers).

Performing follow-up 2D-Echocardiography after 1 month after primary PCI including the same parameters in the first study.

The patients were divided into two groups: improved and non-improved, according to the improvement of LV systolic function measured by GLS. Improvement is defined by increase of GLS $\geq 10 \%$.

\section{Statistical Analysis}

Data were analyzed using Statistical Program for Statistical Program for Social Science (SPSS) version 23.0 for windows (SPSS Inc., Chicago, IL, USA). Quantitative data were expressed as mean \pm standard deviation (SD). Median and range (minimum - maximum) were also calculated for quantitative data. Qualitative data were expressed as frequency and percentage. Mann Whitney U test is used to compare differences between two independent groups when the dependent variable is continuous, but not normally distributed. Chi-square (X2) test also called Pearson's chi-square test or the chi-square test of association is used to discover if there is a relationship between two categorical variables. 


\section{Results}

The mean age of the whole population was $52.82 \pm 10.74$ years with maximal age of 73 years and minimal age of 31 years. While as regard gender, of the 50 patients, there were 45 males $(90 \%)$ and 5 females (10\%). Dyslipidemia was the most prominent risk factor; 26 patients $(52 \%)$. Eight patients (16\%) were hypertensive, while 42 patients $(84 \%)$ were not.
Regarding the glycemic state, 12 patients only (24\%) were diabetic while 38 were non-diabetic representing (76\%). Smoking was risk factor in 24 patients $(48 \%)$ vs. 22 nonsmokers $(44 \%)$ vs. 4 patients $(8 \%)$ are ex-smokers. Eleven patients $(22 \%)$ have positive family history of CAD. Distribution of cardiovascular risk factors in the study population are summarized in Table 1.

Table 1. Distribution of cardiovascular risk factors in study population.

\begin{tabular}{lllll}
\hline & Àll Patients (n=50) & Improvers (n=27) & Non improvers (n=23) & P value \\
\hline Age & $52.82 \pm 10.74$ & $52.19 \pm 9.86$ & $53.57 \pm 11.88$ & 0.655 \\
Gender & & & \\
Male & $45(90 \%)$ & $24(88.9 \%)$ & $21(91.3 \%)$ & 1.000 \\
Female & $5(10 \%)$ & $3(11.1 \%)$ & $2(8.7 \%)$ & 0.711 \\
Hypertension & $8(16 \%)$ & $5(18.5 \%)$ & $3(13 \%)$ & 0.325 \\
Diabetes Mellitus & $12(24 \%)$ & $5(18.5 \%)$ & $7(30.4 \%)$ & 0.346 \\
Dyslipidemia & $26(52 \%)$ & $11(40.7 \%)$ & $15(65.2 \%)$ & 0.658 \\
Family History & $11(22 \%)$ & $6(22.2 \%)$ & $5(21.7 \%)$ & \\
Smoking & & & & \\
Non smoker & $22(44 \%)$ & $14(51.9 \%)$ & $8(34.8 \%)$ & 0.396 \\
Smoker & $24(48 \%)$ & $11(40.7 \%)$ & $13(56.5 \%)$ & \\
Ex-smoker & $4(8 \%)$ & $2(7.4 \%)$ & $2(8.7 \%)$ & \\
\hline
\end{tabular}

Coronary angiography assessment is summarized in Table 2 . Twenty-nine of patients (58\%) had LAD as the IRA, sixteen patients $(32 \%)$ had RCA, four patients $(8 \%)$ had LCX and only one patient $(2 \%)$ had diagonal branch as an IRA. In the endresult, Only five patients (10\%) had TIMI I flow, thirteen (26\%) had TIMI II flow and thirty-two (64\%) had TIMI III flow.

Table 2. Distribution of the studied population according to coronry angiography assessment and Cardic enzymes level.

\begin{tabular}{llll}
\hline & All Patients & Improvers $(\mathbf{n}=27)$ & Non improvers (n=23) \\
\hline $\begin{array}{l}\text { Symptoms to balloon time }(\mathrm{min}) \\
\text { IRA }\end{array}$ & $191(145,264)$ & $189(142,259)$ & $185(145,261)$ \\
LAD & $29(58 \%)$ & $15(55.6 \%)$ & $14(60.9 \%)$ \\
RCA & $16(32 \%)$ & $9(33.3 \%)$ & $7(30.4 \%)$ \\
Diagonal & $1(2 \%)$ & 0 & $1(4.3 \%)$ \\
LCX & $4(8 \%)$ & $3(11.1 \%)$ & $(4.3 \%)$ \\
TIMI flow & $5(10 \%)$ & $2(7.4 \%)$ & $3(13 \%)$ \\
I & $13(26 \%)$ & $7(25.9 \%)$ & $6(26.1 \%)$ \\
II & $32(64 \%)$ & $18(66.7 \%)$ & $14(60.9 \%)$ \\
III & $6(2,11)$ & $5.0(3.5-7.0)$ & 0.909 \\
Peak cardic enzymes level & 2645 & 1684.0 & $12.0(10.5-15.5)$ \\
Peak cTn T ( $\mu$ g/L) & $(947,3717)$ & $(1085.5-2104.5)$ & 4336.0 \\
Peak CPK level (U/L) & & $(3890.5-4967.5)$ \\
\hline
\end{tabular}

CPK, creatine phosphokinase; cTnT, cardiac troponin T; LAD, left anterior descending coronary artery; LCX, left circumflex; Min, minute; PCI, percutaneous coronary intervention; RCA, right coronary artery; TIMI, thrombolysis in myocardial infarction.

Baseline echocardiographic characteristics are summarized in Table 3. Mean LV end-diastolic diameter is $52.44 \pm 7.61$ $\mathrm{mm}$, Mean LV end-systolic diameter is $39.30 \pm 7.26$, and mean LVEF was $48.84 \pm 10.11$. Mean GLPSS at baseline was $213.7+3.3 \%$. Twenty-one patients $(42 \%)$ had normal LV diastolic function, twenty-nine patients $(58 \%)$ had $\mathrm{LV}$ diatolic dysfunction (18\% had grade I, $32 \%$ had grade II and
$8 \%$ hade grade III LV diastolic dysfunction).

During follow-up, Ejection fraction and mean GLPSS increased from $48.84 \pm 10.11$ and $-11.6 \pm 4.40$ at baseline to $49.60 \pm 9.79$ and $-12.82 \pm 4.30$ respectively at 1 month follow-up with no significant difference as regard LV dimensions and diastolic function.

Table 3. Echocardiographic data in our study population.

\begin{tabular}{lllc}
\hline & All Patients $(\mathbf{n = 5 0})$ & Improvers $(\mathbf{n}=\mathbf{2 7})$ & Non-improvers $(\mathbf{n}=\mathbf{2 3})$ \\
\hline LV dimensions & & & \\
LVEDD & $52.44 \pm 7.61$ & $51.44 \pm 6.13$ & $53.60 \pm 9.04$ \\
LVESD & $39.30 \pm 7.26$ & $38.04 \pm 4.93$ & $40.78 \pm 9.18$ \\
LVEF (simpson) & & & 0.355 \\
Early & $48.84 \pm 10.11$ & $50.19 \pm 6.42$ & $47.26 \pm 13.20$ \\
Late & $49.60 \pm 9.79$ & $50.81 \pm 5.78$ & $48.17 \pm 13.04$ \\
\hline
\end{tabular}




\begin{tabular}{lllll}
\hline & All Patients $(\mathbf{n}=\mathbf{5 0})$ & Improvers $(\mathbf{n}=\mathbf{2 7})$ & Non-improvers $(\mathbf{n}=\mathbf{2 3})$ & P value \\
\hline LV diastolic function & & & & \\
Normal & $21(42 \%)$ & $16(59.3 \%)$ & $5(21.7 \%)$ & $4(17.4 \%)$ \\
Grade I & $9(18 \%)$ & $5(18.5 \%)$ & $10(43.5 \%)$ & 0.012 \\
Grade II & $16(32 \%)$ & $6(22.2 \%)$ & $4(17.4 \%)$ & \\
Restrictive & $4(8 \%)$ & 0 & & \\
\hline
\end{tabular}

LVEDD, left ventricular end-diastolic diameter; LVEF, left ventricular ejection fraction; LVESD, left ventricular end-systolic diameter.

Subsequently, the study population was divided in two groups: non-improvers vs. improvers. With improvement defined as $(\geq 10 \%$ increase of GLPSS). The clinical and echocardiographic characteristics of these patients are summarized in Tables 1-3. Comparison between the two studied groups according to GLS is summarized in Table 3. Over half the patients (54\%) improved within 1 month followup, where $46 \%$ did not increase $\geq 10 \%$ in GLPSS during 1 month follow-up after AMI. Non-improvers were more likely to have higher peak cardiac enzymes [4336.0 (3890.5-4967.5) U/L vs. $1684.0(1085.5-2104.5) \mathrm{U} / \mathrm{L}, P<0.001]$ for peak creatine phosphokinase and $[12.0(10.5-15.5) \mathrm{mg} / \mathrm{L}$ vs. 5.0 $(3.5-7.0) \mathrm{mg} / \mathrm{L}, P<0.001]$ for peak cardiac troponin $\mathrm{T}$ level. Furthermore, nonimprovers had diastolic dysfunction and lower GLPSS $(-10.96 \pm 3.85$ vs. $-12.27 \pm 3.73, P=0.047)$. No significant differences were observed in LVEF between improvers and nonimprovers (50.19 \pm 6.42 vs. 47.26 \pm 13.20 , $P=0.340)$. Improved group showed statistically significant improvement in $\mathrm{EF}$ (from $50.19 \pm 6.42$ to $50.81 \pm 5.78 P<0.016$ ) in the baseline and 1 month follow up respectively.

Peak cardiac troponin T level (OR $0.21,95 \%$ CI $0.14-0.32$, $P$ 0.001), Peak creatine phosphokinase level, diastolic function $\geq$ Grade I (OR 4.71, 95\% CI 1.13-19.63, $P$ 0.012), and baseline GLPSS (OR 1.26, 95\% CI 1.08-1.46, $P$ 0.003) were independent predictors of improvement of LV function.

\section{Discussion}

The major findings of this study were: (i) During 1 month follow-up of patients after AMI, 54\% of patients showed improvement of LV function (defined as $\geq 10 \%$ increase of GLS). (ii) The patients who showed improvement of GLS were associated with improvement of EF (using simpson method). (iii) Peak cardiac troponin T, Peak CPK levels, LV diastolic function, and baseline GLS were independent predictors of improvement of LV function during follow-up. Baseline GLPSS provided incremental value to traditional parameters for the prediction of recovery of LV function.

\subsection{Quantification of Left Ventricular Systolic Function}

Previous studies have demonstrated that GLPSS correlated well with LVEF in the overall population and good intra- and inter-observer agreements have been shown. Delgado V et al. reported in a study of 1060 patients that LV GLS was independently related to all-cause mortality and combined end point and had prognostic incremental value over other well-established clinical and ECG predictors [3].

Table 4. Comparison between the two studied groups according to GLPSS.

\begin{tabular}{|c|c|c|c|c|c|}
\hline & GLS & All patients $(n=50)$ & Improved $(n=27)$ & Non-improved $(n=23)$ & $\mathbf{p}$ \\
\hline \multirow{3}{*}{ LAX } & Early & $-10.40(-8.5--14.1)$ & $-12.20(-8.5--14.5)$ & $-9.80(-8.4--12.9)$ & 0.340 \\
\hline & Late & $-11.30(-9.4--15.6)$ & $-13.40(-10.4--16.5)$ & $-10.25(-8.6--13.9)$ & 0.054 \\
\hline & $\mathrm{P} 1$ & $<0.001^{*}$ & $<0.001^{*}$ & $<0.001^{*}$ & \\
\hline \multirow[t]{2}{*}{$\mathrm{A} 4 \mathrm{C}$} & Late & $-12.97 \pm 4.31$ & $-14.26 \pm 3.63$ & $-11.40 \pm 4.63$ & $0.019 *$ \\
\hline & $\mathrm{P} 1$ & $<0.001 *$ & $<0.001^{*}$ & $<0.001 *$ & \\
\hline \multirow{2}{*}{$\mathrm{A} 2 \mathrm{C}$} & Early & $-11.82 \pm 4.87$ & $-12.36 \pm 4.17$ & $-11.15 \pm 5.64$ & 0.393 \\
\hline & $\mathrm{P} 1$ & $<0.001 *$ & $<0.001^{*}$ & $0.001 *$ & \\
\hline \multirow{3}{*}{ Average } & Early & $-11.6 \pm 4.40$ & $-12.27 \pm 3.73$ & $-10.96 \pm 3.85$ & $0.047 *$ \\
\hline & Late & $-12.82 \pm 4.30$ & $-13.96 \pm 3.54$ & $-11.21 \pm 3.98$ & $0.038 *$ \\
\hline & $\mathrm{P} 1$ & $<0.001 *$ & $<0.001^{*}$ & $<0.001 *$ & \\
\hline
\end{tabular}

A2C, apical 2 chamber; A4C, apical 4 chamber; LAX, long axis view.

\subsection{Evolution of LV Function After Acute STEMI}

Left ventricular dysfunction after AMI is caused by a combination of myocardial stunning and necrosis. Since residual LV function is of important prognostic value for long-term survival, information on the evolution of LV function is important. In the current study, most patients (54\%) showed improvement of LV function which was defined as $\geq 10 \%$ increase of GLS. It can be explained by myocardial stunning. Stunning is defined as post-ischaemic reversible dysfunction of the myocardium and recovers during a period of weeks to months [4].

M. Louisa Antoni et al. investigated the recovery of LV function using GLPSS in 341 patients with echocardiography performed on Day 1 then at 3,6 and 12 months after AMI. They reported the same recovery rate of LV function (54\%) at 3 months follow-up with higher recovery rate of $\mathrm{LV}$ function (72\%) during 1 year follow-up [5]. This study was the first to evaluate the time course of LV systolic function, quantified with GLPSS during 1 year follow-up after AMI. In that study, It was notet that only $18 \%$ of the patients revealed 
late improvement of LV systolic function. This finding implies that, although the majority of LV recovery occurs in the first weeks after revascularization, improvement can continue for up to 6 months or more. The comparison of patients with early and late improvement of $\mathrm{LV}$ function demonstrated no significant differences in baseline characteristics.

In another prospective study including 249 patients, Solomon et al. investigated the recovery of LV function with echocardiography performed on Days 1, 14, and 90 after AMI [6]. The authors reported a higher recovery rate of LV function in $58 \%$ of patients at 90 days, where most of the changes occurred in the first 14 days.

Also, Parodi et al. investigated the recovery of $\mathrm{LV}$ function in a prospective study of 228 patients with echocardiography performed within $24 \mathrm{~h}$, at 1 and 6 months after AMI and reported a recovery rate of $58 \%$ after 6 months (defined as $\geq 10 \%$ increase of LVEF) [7]. Interestingly, The 5-year cardiac death rate was significantly lower in patients with recovery of LV function when compared with patients without recovery ( 8 vs. $18 \%$, respectively, $P=0.024)$, but with no differences observed in the probability of survival between patients with early recovery (1 month) and late recovery (6 months) of LV function ( 91 vs. $93 \%, P=0.92$ ).

Mollema et al. investigated the recovery of LV function in a prospective study of 147 patients with echocardiography performed within $48 \mathrm{~h}$ and at 1 year after AMI and reported a lower recovery rate of $48 \%$ after 12 months (defined as $\geq 5 \%$ increase of LVEF) [8].

\subsection{Prediction of Improvement of LV Function After Acute STEMI}

The results of our study support previous studies [11] that peak cardiac enzymes level is strongly related to the recovery of LV function and is more important than other early clinical measures. In a study of 855 patients, Ohman EM et al. demonstrated that cardiac troponin $\mathrm{T}$ level is a powerful, independent risk marker in patients who present with AMI [9]. Also, S D Solomon et al. demonstrated in a study of 352 patients that a lower peak level of creatine kinase, an estimate of the extent of necrosis, is independently predictive of recovery of function [6].

In addition to peak cardiac enzymes level, diastolic dysfunction was a significant predictor of recovery of LV function. In agreement with our results, Poulsen et al. performed a study of 58 patients about prognostic implications of LV diastolic function in first AMI. The patients were studied serially within 1 hour and at days 5, 90, and 360. They demonstrated that the presence of early diastolic dysfunction after AMI was associated with an increased risk for $\mathrm{LV}$ remodelling and development of congestive heart failure [11].

Furthermore, a meta-analysis (MeRGE-AMI) of 12 prospective post-infarction trials (3739 patients) showed that the presence of restrictive filling pattern (defined as high E/A ratio and/or shortened deceleration time) after AMI was a strong predictor of mortality [10].

The last prognostic predictor identified at that study is baseline GLPSS. Baseline GLPSS provided incremental value to traditional parameters for the prediction of recovery of LV function. M. Louisa Antoni et al. demonstrated that Non improvers were more likely to have lower GLPSS than improvers $(-13.2 \pm 3.0$ vs. $-14.1 \pm 3.1 \%$, $P=0.04$ ) and considered as independent predictor of improvement of LV function during follow-up [5]. Also, Mollema et al. demonstrated that baseline GLS was a predictor for change in LV ejection fraction at 1-year follow-up [7].

In contrary to our results, $M$. Louisa Antoni et al. demonstrated that the LAD as culprit vessel was identified as independent predictors of recovery of LV function [5]. Also, Parodi $\mathrm{G}$ et al. demonstrated that time from symptom onset to reperfusion and female gender were Independent predictors of LV functional recovery [8].

\section{Conclusion}

Improvement of LV systolic function after AMI occurred in 54\% during 1 month follow-up. Peak cardiac troponin $\mathrm{T}$ level, and diastolic function, GLS measured at baseline was an independent predictor of recovery of LV function. Although changes in GLS during follow-up were modest, GLS provided incremental value to traditional parameters for the prediction of improvement of LV function. Quantification of GLPSS at baseline may be of important value for the prediction of recovery of LV function in patients after Acute STEMI.

\section{Conflict of Interest}

On behalf of all authors, the corresponding author states that there is no conflict of interest.

\section{References}

[1] Oana Mirea, Jurgen Duchenne, Jens-Uwe Voigt. Recent advances in echocardiography: strain and strain rate imaging. F1000Res. 2016; 5: F1000 Faculty Rev-787.

[2] Lang RM, Badano LP, Mor-Avi V, et al.: Recommendations for cardiac chamber quantification by echocardiography in adults: an update from the American Society of Echocardiography and the European Association of Cardiovascular Imaging. Eur Heart J Cardiovasc Imaging. 2015; 16 (3): 233-70. 10.1093/ehjci/jev014.

[3] Delgado V, Mollema SA, Ypenburg C, Tops LF, van der Wall EE, Schalij MJ, Bax JJ. Relation between global left ventricular longitudinal strain assessed with novel automated function imaging and biplane left ventricular ejection fraction in patients with coronary artery disease. J Am Soc Echocardiogr 2008; 21: 1244-1250.

[4] Adam K. McDiarmid1, Pierpaolo Pellicori2, John G. Cleland2, and Sven Plein. European Heart Journal (2017) 38, 942-954. doi: 10.1093/eurheartj/ehw140. 
[5] M. Louisa Antoni, Sjoerd A. Mollema1, Jael Z. Atary, C. Jan Willem Borleffs, Eric Boersma, Nico R. L. van de Veire, Eduard R. Holman, Ernst E. van der Wall, Martin J. Schalij, and Jeroen J. Bax. Time course of global left ventricular strain after acute myocardial infarction. European Heart Journal (2010) 31, 2006-2013 doi: 10.1093/eurheartj/ehq198.

[6] Solomon SD, Glynn RJ, Greaves S, Ajani U, Rouleau JL, Menapace F, Arnold JM, Hennekens C, Pfeffer MA. Recovery of ventricular function after myocardial infarction in the reperfusion era: the healing and early afterload reducing therapy study. Ann Intern Med 2001; 134: 451-458.

[7] Parodi G, Memisha G, Carrabba N, Signorini U, Migliorini A, Cerisano G, Antoniucci D. Prevalence, predictors, time course, and long-term clinical implications of left ventricular functional recovery after mechanical reperfusion for acute myocardial infarction. Am J Cardiol 2007; 100: 1718-1722.19.

[8] Mollema SA, Delgado V, Bertini M, et al: Viability assessment with global left ventricular longitudinal strain predicts recovery of left ventricular function after acute myocardial infarction. Circ Cardiovasc Imaging 2010; 3: 15-23.

[9] Ohman EM, Armstrong PW, Christenson RH, et al. Cardiac troponin $\mathrm{T}$ levels for risk stratification in acute myocardial ischemia. GUSTO IIA Investigators. N Engl J Med 1996; 335: 1333-1341.

[10] Moller JE, Whalley GA, Dini FL, Doughty RN, Gamble GD, Klein AL, Quintana M, Yu CM. Independent prognostic importance of a restrictive left ventricular filling pattern after myocardial infarction: an individual patient meta-analysis: Meta-Analysis Research Group in Echocardiography acute myocardial infarction. Circulation 2008; 117: 2591-2598.

[11] Poulsen SH, Jensen SE, Egstrup K. Longitudinal changes and prognostic implications of left ventricular diastolic function in first acute myocardial infarction. Am Heart J 1999; 137: 910 918. 